\title{
Using cloud-based mobile technology for assessment of competencies among medical students.
}

Valid, direct observation of medical student competency in clinical settings remains challenging and limits the opportunity to promote performance-based student advancement. The rationale for direct observation is to ascertain that students have acquired the core clinical competencies needed to care for patients. Too often student observation results in highly variable evaluations which are skewed by factors other than the student's actual performance. Among the barriers to effective direct observation and assessment include the lack of effective tools and strategies for assuring that transparent standards are used for judging clinical competency in authentic clinical settings. We developed a web-based content management system under the name Just in Time Medicine (JIT) to address many of these issues. The goals of JIT were fourfold: First, to create a self-service interface allowing faculty with average computing skills to author customizable content and criterion-based assessment tools displayable on internet enabled devices, including mobile devices; Second, to create an assessment and feedback tool capable of capturing learner progress related to hundreds of clinical skills; Third, to enable easy access and utilization of these tools by faculty for learner assessment in authentic clinical settings as a means of just in time faculty development; Fourth, to create a permanent record of the trainees' observed skills useful for both learner and program evaluation. From July 2010 through October 2012, we implemented a JIT enabled clinical evaluation exercise (CEX) among 367 third year internal medicine students. Observers (attending physicians and residents) performed CEX assessments using JIT to guide and document their observations, record their time observing and providing feedback to the students, and their overall satisfaction. Inter-rater reliability and validity were assessed with 17 observers who viewed six videotaped student-patient encounters and by measuring the correlation between student CEX scores and their scores on subsequent standardizedpatient OSCE exams. A total of 3567 CEXs were completed by 516 observers. The average number of evaluations per student was $9.7( \pm 1.8 \mathrm{SD})$ and the average number of CEXs completed per observer was 6.9 ( $\pm 15.8 \mathrm{SD})$. Observers spent less than 10 minutes on $43-50 \%$ of the CEXs, and on $68.6 \%$ of the feedback sessions. A majority of observers (92\%) reported satisfaction with the CEX. Inter-rater 
reliability was measured at 0.69 among all observers viewing the videotapes and these ratings adequately discriminated competent from non-competent performance. The measured CEX grades correlated with subsequent student performance on an end-of-year OSCE. We conclude that the use of JIT is feasible in capturing discrete clinical performance data with a high degree of user satisfaction. Our embedded checklists had adequate inter-rater reliability and concurrent and predictive validity. 
1 Gary S Ferenchick, MD

2 David Solomon, $\mathrm{PhD}$

3 Department of Internal Medicine

4 Division of General Medicine

5 Michigan State University

6 East Lansing, Michigan, USA

7 Corresponding Author:

8 Gary S Ferenchick, MD

9 B 334 Clinical Center

10 Michigan State University

11 East Lansing, Michigan 48824

12 (517) 4323390 (office)

13 Gary.ferenchick@ht.msu.edu 


\section{Introduction}

15 The assessment of the clinical competence of a medical student is challenging. A

16 competency is "... an observable ability of a health professional related to a specific activity that

17 integrates knowledge, skills, values, and attitudes. Since they are observable, they can be

18 measured and assessed." Although seemingly straight forward, competency based education is of

19 limited usefulness in guiding the design and implementation of educational experiences if they

20 are not tied to specific learning objectives.(1) Additionally, learning objectives are of limited

21 usefulness if they are not available to students and faculty when interacting with patients. Finally,

22 observation and assessment help neither students nor patients if they are not captured and

23 documented in a way that facilitates learner specific plans for improvement and excellence. We

24 present a generalizable initiative that makes national curricula functional in local learning

25 environments, and improves and simplifies observation based assessments and performance-

26 based data tracking for faculty and learners.

\section{Materials \& Methods}

\section{Content Manager}

29 We developed a mobile, cloud-based application called just in time medicine (or JIT) that

30 functions effectively on smart phones, tablets and laptop computers. The mobile application is

31 supported by a self-service web-based content management system designed with the explicit aim

32 of enabling users with average computing skills to build their own customizable content,

33 including criterion-based checklists that can then be delivered to any internet enabled device such

34 as a smart phone or tablet.

35 For this project, we utilized nineteen core training problems from the nationally validated 36 Clerkship Directors in Internal Medicine (CDIM) curriculum and combined these training 
37 problems with the observable competencies of communication skills, history taking and physical

38 examination to create problem and task specific checklists. For each assessment, the software

39 calculates the students' performance by determining the percentage of all potential items

40 performed correctly, and an algorithm generated grade of "not done/unsatisfactory", "needs

41 improvement" or "well done" is calculated depending on the percentage of items performed

42 correctly. In general if a student achieved $80 \%$ of the expected items correctly they received a

43 "well done grade"; performing $30-79 \%$ of the expected items resulted in a "needs

44 improvement" grade, and $<30 \%$ an unsatisfactory grade. Figures $1-2$ present screen shots for

45 the process of building checklists using our content manager for the problem altered mental

46 status and the competency history taking. Additionally, Figures 3 - 4 show how the assessment

47 tools are displayed on the user's device. Figures 5 - 7 show the permanent cloud-based reporting

48 options associated with individual assessments. A fully functional version of JIT can be accessed

49 at: $\underline{w w w . j u s t i n t i m e m e d i c i n e . c o m / m o b i l e ; ~} \log$ in username is testuser@journal.com, and the

50 password is test.

\section{Goals and hypotheses}

52

In introducing the JIT in our clerkship, we hypothesized that JIT would: 1) facilitate the

54 direct observation and provision of feedback to trainees on their clinical competencies; 2)

55 generally be accepted by faculty; 3) provide a means for recording the observations of trainee

56 performance, and 4) possess adequate reliability and validity.

57 Setting

58 The College of Human Medicine (CHM) at Michigan State University is a community-

59 based medical school with clinical training in 7 communities throughout Michigan. Between

60 July 2010 and October 2012 we implemented JIT as an integral part of the internal medicine

61 clerkship among 367 students. Each student was required to complete ten directly observed 
62 clinical evaluation exercises (i.e. CEX's) with real patients in authentic clinical settings. A CEX is

63 a short (generally $<20$ minutes) directly observed trainee - patient interaction (e.g. history-

64 taking, examination, counseling etc.); faculty observes rates and provides written comments on

65 the interaction. Students received an orientation to the CEX application and were required to

66 become familiar with the software. Evaluators (attending faculty and residents) received an email

67 on the importance of direct observation and the basic functionality of the CEX application.

68 In general, students chose the patient, problem and competency upon which to be

69 assessed. At the time of the assessment, students handed their mobile device, with the checklists

70 displayed for evaluator use during the assessed interaction. A total of 516 evaluators

71 subsequently used JIT to guide their observations and assessments of students' interacting with

72 patients.

\section{Data Collection}

74 We collected the following data: the specific training problems and competencies

75 observed and assessed by the evaluators, the grades associated with the observation and

76 descriptive data from faculty on the use of JIT. Descriptive data was collected from the faculty

77 via "pull-down" menus located on the last screen of each assessment. A screen shot of the

78 interface is displayed in figure 4.

\section{Reliability and validity assessments}

80 A group of 17 evaluators, 9 internal medicine residents and 8 general internist faculty

81 members viewed and rated six scripted videotaped encounters using JIT. Each case was scripted

82 for both satisfactory and unsatisfactory performance. These cases have been previously validated

83 by Holmboe as representing levels of competence which range from unequivocally poor to

84 satisfactory.(2) The sample of raters reflected the number we could reasonably obtain given our

85 small general internal medicine faculty and residency program. We felt it was adequate to provide 
86 a stable estimate of the inter rater reliability of the assessment process. We calculated the inter

87 rater reliability using a formula developed by Ebel and implemented using software developed by

88 one of the authors. $(3,4)$ All other statistical analyses were performed with SPSS version 21.

\section{Results}

\section{Number and types of evaluations}

91 Five hundred sixteen evaluators used the application to assess 367 students for a total of

923567 separate assessments. The number of CEX's completed per student was $9.7( \pm 1.8)$ and the

93 average number of CEX's completed per faculty was $6.9( \pm 15.8)$. The average number of

94 training problems a student was assessed on was 6.7; of the three competency domains of

95 communication skills, history taking, and physical examination $68 \%$ of the students had at least

96 one evaluation in each of the three categories.

97 In terms of the grades, time variables and satisfaction, $\sim 83 \%$ of the encounters were

98 associated with a "well done" grade, and on average students were credited with performing

$9986 \%$ of the items correctly. (Figure 8) Between $43-50 \%$ of the CEX assessments took $<10$

100 minutes as estimated by the faculty, and in $\sim 69 \%$ of the encounters feedback was estimated to

101 occur in less than 10 minutes. In $92 \%$ of the encounters, faculty rated that they were either

102 satisfied or highly satisfied with the CEX.

103 The estimated inter-rater reliability of a single rater observing the videotaped encounters

104 was 0.69 (slightly higher for faculty at 0.74 vs. residents at .64). In judging the same simulated

105 patient case scripted to be satisfactory and non-satisfactory, the residents and faculty using JIT

106 discriminated between the satisfactory and non-satisfactory performance. The mean number of

107 items checked for the videotapes scripted for unsatisfactory performance was 35\% vs. 59\% for

108 those scripted for more satisfactory performance. We believe this provides evidence supporting

109 the construct validity of JIT. 
111 between a "gateway" performance assessment examinations taken by 282 students at the end of

112 their third year required clerkships with the CEX assessments obtained by JIT. There was a small

113 (but statistically significant $0.144, \mathrm{p}=.008$ ) correlation between students' CEX scores and

114 communications skills in the gateway performance assessment exam.

\section{Discussion}

116 Although national learning objectives have been published for all core clerkships, their

117 usefulness for assessing learning outcomes has been limited. As an example, the core competency

118 gathering essential and accurate information seems relatively straight forward. However, when

119 applied to a single condition such as chronic obstructive pulmonary disease, there are at least 28

120 specified clinical tasks related to history taking and performing a physical examination that a

121 student should demonstrate to meet the expected outcomes as defined in the Clerkship Directors

122 in Internal Medicine (CDIM) curricular objectives for that problem. Of these 28, how many will a

123 faculty evaluator remember when assessing the student? More importantly how many can they

124 remember and what level of consistency will there be among preceptors providing feedback to

125 students?

126 If we take almost any clinical skill and start to dissect it, we find very quickly that

127 existing human memory is insufficient in recalling all of the explicit steps related to potentially

128 hundreds of conditions that help frame the expected outcomes of a trainee's educational

129 experience and curricula. As the expectations for assessment of discrete competencies increases,

130 the evaluation burden for educators, students and administrators becomes progressively more

131 educationally incomplete and logistically unmanageable. 
The inability of faculty to remember and accurately assess for outcomes related to

133 potentially hundreds of discrete educational objectives while evaluating trainees in clinical

134 settings is one of the major reasons faculty have a hard time reliably discriminating unsatisfactory

135 from satisfactory performance, as has been noted by many authors over the past decade using

136 paper-based systems. $(2,5)$ For example, in a study of mini-CEX evaluations among 300 medical

137 students, Hill noted that problems existed "in trying to ensure that everyone was working to the

138 same or similar standards."(6) In another study of 400 mini-CEX assessments, Fernando

139 concluded faculty evaluators were unsure of the level of performance expected of the learners.(7)

140 Hasnain noted that poor agreement among faculty evaluating medical students on a Family

141 Medicine clerkship was due to the fact that "Standards for judging clinical competence were not

142 explicit”.(8) In a randomized trial of a faculty development effort, Holmboe studied the accuracy

143 of faculty ratings by having them view videotaped trainee-patient encounters that were scripted to

144 portray three levels of proficiency; unsatisfactory, marginal or satisfactory. Faculty viewing the

145 exact same encounter varied widely in their assessment of trainee competence, with ratings from

146 unequivocally unsatisfactory (CEX scores of scores $1-3)$ to unequivocally superior (CEX scores

147 of $7-9)$, regardless of whether the video was scripted to be unsatisfactory or not. After an

148 intensive 4 day faculty development workshop in which participants were tasked with developing

149 a shared mental model of what specific competencies should look like, problems still existed

150 among faculty in discriminating satisfactory from unsatisfactory performance in these scripted

151 encounters.(2)

152 Kogan noted that in the absence of easily accessible frameworks, faculty evaluators

153 default back to a myriad of highly variable evaluation strategies including such idiosyncratic

154 features as instinct, "gut feelings", "unsubstantiated assumptions" and the faculty members'

155 emotional response to providing feedback. What she also noted was that faculty raters commonly 
156 fail to use existing frameworks or external standards in guiding their evaluations of trainees, thus

157 explaining much of the well-recognized problems with poor validity and inter-rater reliability

158 associated with clinical evaluations.(5)

159 Given these realities, it is not surprising that medical trainees commonly do not view the

160 feedback received from faculty as credible nor influential in learning, especially if the feedback

161 was not immediate and tied to the trainees' clinical work-place performance. (9) Enhancing the

162 effectiveness of clinical assessments, the delivery of feedback related to learning objectives and

163 the creation of better systems for documenting faculty observations are commonly cited needs in

164 medical education. $(8,10-13)$

165 Given these and other trends, systems that are capable of disseminating curricular

166 objectives to students and faculty and which also enable criterion-based assessment have become

167 key educational needs. We believe that cloud-based technology, appropriately applied to

168 maximize efficiency, can contribute to optimizing the learning environment by directly aligning

169 learning objectives from national disciplinary curricula with assessment tools for use by students

170 and faculty anywhere and anytime, especially at the bedside.

171 In our first feasibility study, we demonstrated our ability to deliver national educational

172 objectives published by the CDIM to electronic hand-held personal digital assistants (PDAs) such

173 as Palm ${ }^{\circledR}$ and PocketPC ${ }^{\circledR}$ devices.(14) In a second feasibility study, we subsequently

174 demonstrated this system could be used to deliver and successfully implement competency-based

175 checklists for student assessment related to the CDIM curricular objectives using PDAs.(15) Data

176 from these studies helped us determine that the distribution and use of curricular objectives and

177 related assessment tools by students and faculty in our geographically dispersed medical school

178 could be facilitated with just in time mobile technology. Importantly, we also determined that 
179 students and preceptors valued the fact that the content and expected competencies were

180 transparent and such transparency facilitated learner assessment.(15) However, technical issues

181 with PDAs -- such as lack of direct internet connection and the requirement to "synchronize" data

182 from PDAs to the web using desktop computers -- limited the practicality of PDA based

183 assessment; a process that is not needed with contemporary internet enabled devices such as

184 iPads, iPhones and other smart phones. These devices have become almost ubiquitous in the past

185 four years and we have leveraged this trend to evolve JIT to a platform-neutral Cloud-based

186 system. The displayed assessment tools function like an "application" on mobile devices, but are

187 device-agnostic in that they functions on all internet-enabled devices, including smart phones.

188 Out study, like most others, have several inherent limitations. First, this is a single

189 institution study and these results may not be generalizable. Future studies should focus on the

190 use of this technology in other settings. Second, establishing the reliability of all of the

191 customized checklists within the CEX application is needed, as is establishing its reliability in

192 real clinical settings such as the hospital wards. Third, we have not established the validity of the

193 electronic grading algorithm. Fourth, like many tools for direct observation, we have not

194 established the effect of this tool on learning nor the transfer of acquired clinical skills to other

195 areas, or the effect that such direct observation has on the most important outcome of patient 196 care.

\section{Conclusions}

198 We have established that just in time Cloud-based mobile technology has great potential

199 in competency-based medical education. Although not an objective of this study, we believe such

200 technology holds great promise for use in authentic clinical settings for measuring student

201 achievement related to educational milestones. Additionally, given the time and cost constraints 
202 associated with traditional faculty development efforts, we believe that systems such as JIT have 203 great potential in operationalizing "just in time" faculty development. 
205 1. Whitcomb ME. More on competency-based education. Acad Med. 2004;79(6):493-4.

206 2. Holmboe ES, Hawkins RE, Huot SJ. Effects of training in direct observation of medical 207 residents' clinical competence: a randomized trial. Ann Intern Med. 2004;140(11):874-81.

208 3. RL E. Estimation of the reliability of ratings. Psychometrika. 1951;16:407-24.

209 4. Solomon DJ. The rating reliability calculator. BMC Med Res Methodol. 2004;4:11.

210 5. Kogan JR, Conforti L, Bernabeo E, Iobst W, Holmboe E. Opening the black box of clinical skills assessment via observation: a conceptual model. Med Educ. 2011;45(10):1048-60.

6. Hill F, Kendall K, Galbraith K, Crossley J. Implementing the undergraduate mini-CEX: a tailored applicationroach at Southampton University. Med Educ. 2009;43(4):326-34.

7. Fernando N, Cleland J, McKenzie H, Cassar K. Identifying the factors that determine feedback given to undergraduate medical students following formative mini-CEX assessments. Med Educ. 2008;42(1):89-95.

8. Hasnain M, Connell KJ, Downing SM, Olthoff A, Yudkowsky R. Toward meaningful evaluation of clinical competence: the role of direct observation in clerkship ratings. Acad Med. 2004;79(10 Suppl):S21-4.

9. Watling C, Driessen E, van der Vleuten CP, Lingard L. Learning from clinical work: the roles of learning cues and credibility judgements. Med Educ. 2012;46(2):192-200.

10. Howley LD, Wilson WG. Direct observation of students during clerkship rotations: a multiyear descriptive study. Academic medicine : journal of the Association of American Medical Colleges. 2004;79(3):276-80. and user satisfaction with a PDA-based mini-CEX to evaluate the clinical skills of thirdyear medical students. Teaching and learning in medicine. 2007;19(3):271-7. 
229 12. Hauer KE, Kogan JR. Realising the potential value of feedback. Med Educ.

$230 \quad 2012 ; 46(2): 140-2$.

231 13. Whitcomb ME. Competency-based graduate medical education? Of course! But how should competency be assessed? Acad Med. 2002;77(5):359-60.

233 14. Ferenchick G, Fetters M, Carse AM. Just in time: technology to disseminate curriculum 234 and manage educational requirements with mobile technology. Teach Learn Med. 2008;20(1):44-52.

15. Ferenchick GS, Foreback J, Towfiq B, Kavanaugh K, Solomon D, Mohmand A. The implementation of a mobile problem-specific electronic CEX for assessing directly 238 observed student-patient encounters. Med Educ Online. 2010;15. 


\section{Figure 1}

\section{Step 1. Content Manager for Development of Assessment Tools}

Using simple interfaces, faculty adds content (e.g. the problem altered mental status) and the specific competency to be assessed (e.g. history taking)

\section{Assessment Admin - Groups}

Organizational Unit: Internal Medicine (MED 608)

\begin{tabular}{lllll} 
& & & \multicolumn{1}{c}{ Competency } & Combo Name \\
\hline Select & Abdominal Pain & Communication Skills & Case Pres & Delete \\
\hline Select & Abdominal Pain & History Taking Skills & Abd Pain: History & Delete \\
\hline Select & Abdominal Pain & Physical Exam Skills & Physical Exam & Delete \\
\hline Select & Altered Mental Status & Communication Skills & Case Pres & Delete \\
\hline Select & Altered Mental Status & History Taking Skills & History & Delete \\
\hline Select & Altered Mental Status & Physical Exam Skills & Physical Exam & Delete \\
\hline Select & Anemia & Communication Skills & Case Pres & Delete \\
\hline Select & Anemia & History Taking Skills & History & Delete \\
\hline Select & Anemia & Physical Exam Skills & Abnormalities & Delete \\
\hline Select & Anemia & Physical Exam Skills & Physical & Delete \\
\hline Select & Back pain & Communication Skills & Case Pres & Delete \\
\hline Select & Back pain & Communication Skills & Communication & Delete \\
\hline Select & Back pain & History Taking Skills & History & Delete \\
\hline Select & Back pain & Physical Exam Skills & Abnormalities & Physical Exam \\
\hline Select & Back pain & Physical Exam Skills &
\end{tabular}




\section{Figure 2}

\section{Step 2. Content Manager for Development of Assessment Tools}

Using the self-service web-based content management system, faculty then adds assessment questions reflecting specific criterion-based outcomes (e.g. The student started the interview with open-ended questions)

\begin{tabular}{|c|c|c|c|c|c|}
\hline Details & Line Items & Grading Tiers & Taxonomies & & \\
\hline & & & Line Item Name & Ordir & \\
\hline \multicolumn{2}{|l|}{ Selected } & & Dr-Pt Comm / Characterizes Sx & 1 & Delete \\
\hline \multicolumn{2}{|l|}{ Select } & & Assoc Sx's \& Delirium & 2 & Delete \\
\hline \multicolumn{2}{|l|}{ Select } & & Past $\mathrm{Hx} / \mathrm{Habits}$ & 3 & Delete \\
\hline \multicolumn{2}{|l|}{ Select } & & Medications & 4 & Delete \\
\hline \multicolumn{3}{|c|}{ Add New Line ltem } & & & \\
\hline etails & rading Tiers & & & & \\
\hline
\end{tabular}

Line ltem Name

Dr-Pt Comm / Characterizes Sx

Ordinal

I

Checkmark Type

Yes/No

Description

Pt-Centered interviewing: The student specifically:

$\rightarrow$. Started interview with open-ended questions

$\rightarrow$. Asked how the patient is coping

$\rightarrow$ • Demonstrated concern for the patient

$\rightarrow$ - Responded with at least I empathic statement

- Asked the patient if they had any questions

Characterizes CC: Student asks about, elicits or establishes:

- Hx of similar problem

- Onset (probes for evidence of an acute change)

- Activity when mental status changed

- Pattern: intermittent or constant

- Pattern: change in severity (increase or decrease)

- Recent Trauma 


\section{Figure 3}

Criterion-based assessment for altered mental status and history-taking as displayed on the mobile device for use anytime and anywhere

Screen shot A displays how the specific checklist is accessed on the device; screen shot B displays the criterion-based tasks, which are defaulted to No change to Yes (screen shot C) once the task is completed by the learner. Screen shot D displays the alogrithm generated grade

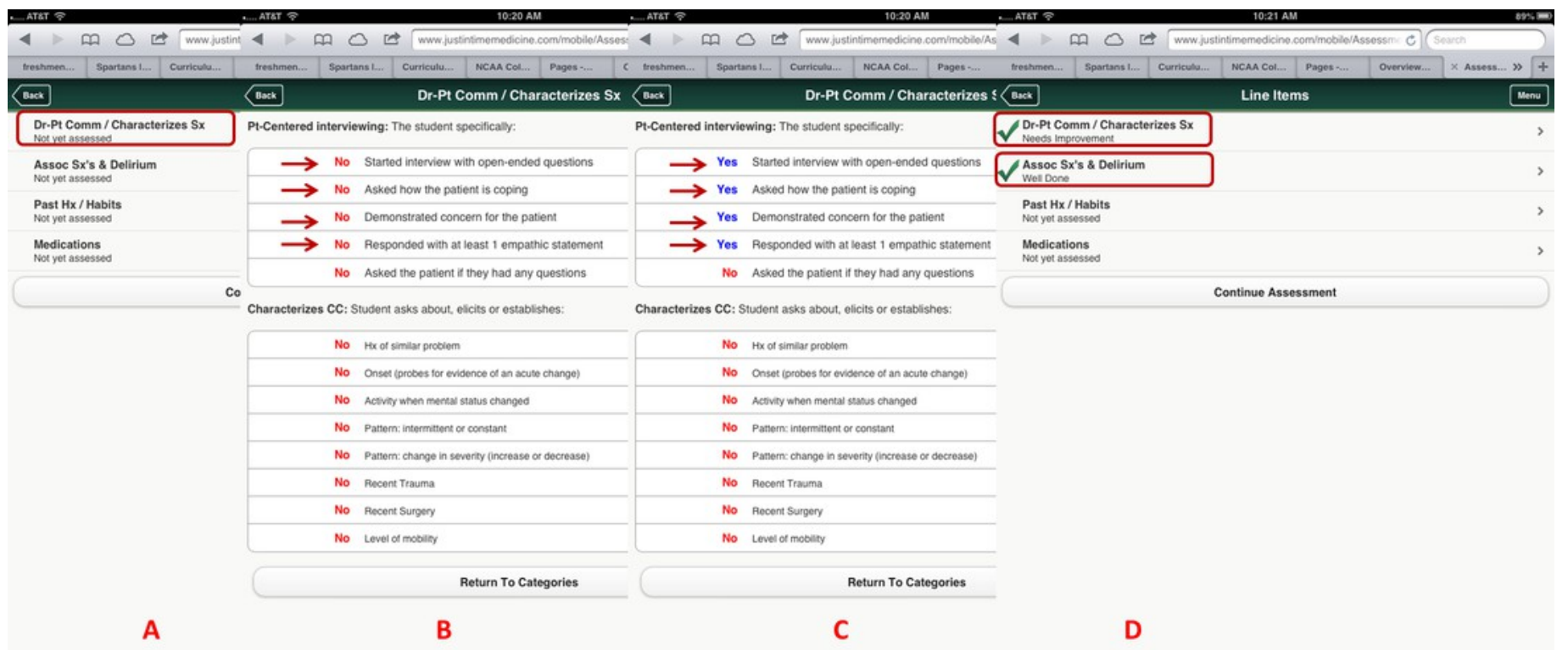




\section{Figure 4}

Evaluator information is collected using simple interfaces on the device after the assessment is completed, including open-ended qualitative comments.

Faculty enters information concerning their observation (screen shot A), their feedback and action plans (screen shot B). A color coded competency registry is displayed on the learners device (screen shot C). Note in screen shot B, the evaluator has the option to have an email link sent to him/her to complete the qualitative assessment at a later time. All evaluations become part of the learners cloudbased permanent record.

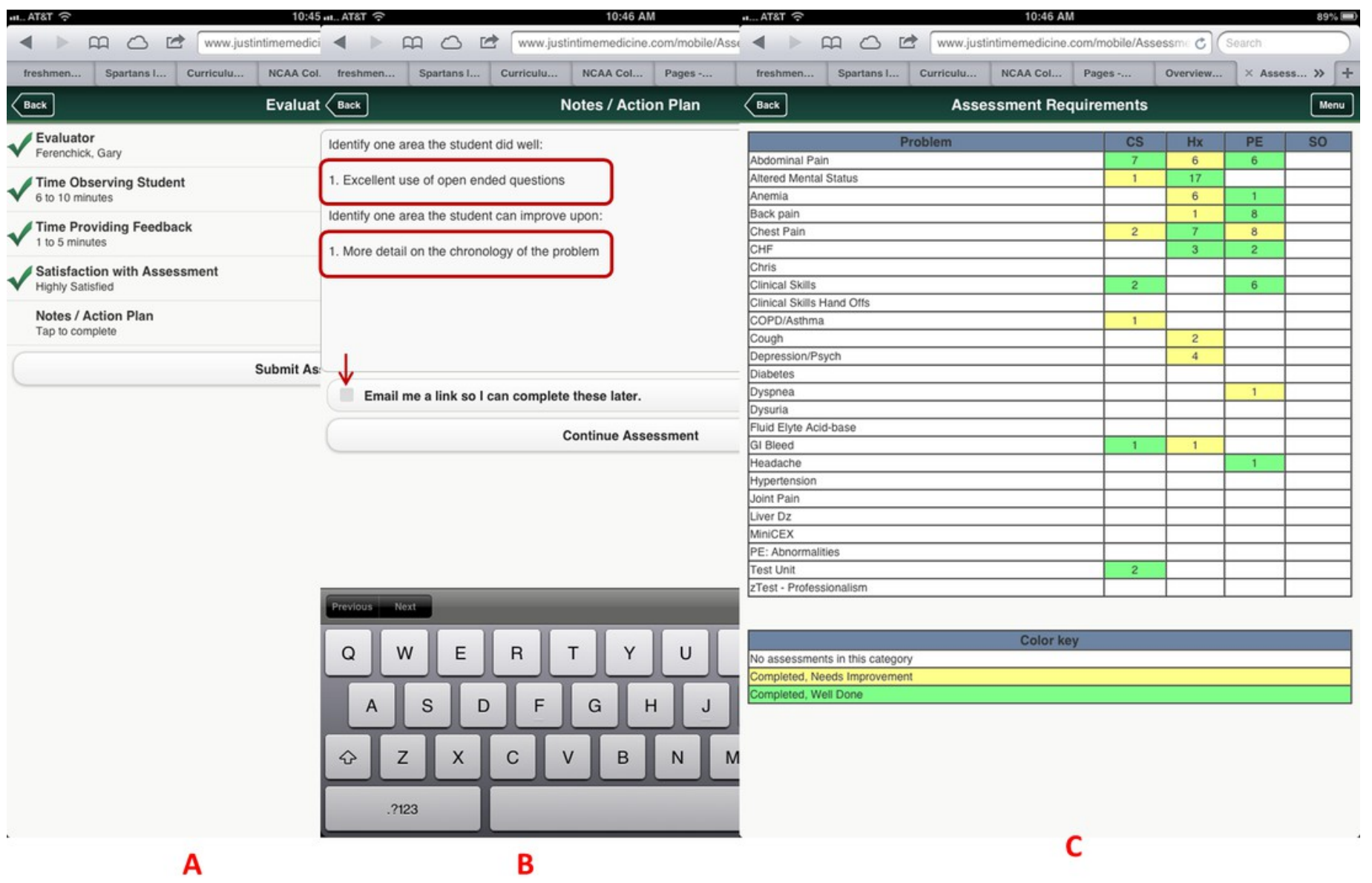




\section{Figure 5}

\section{Detailed Cloud-based Reporting Options}

One of the web-based permanent records of the students' performance; displaying the item(s) assessed, the percentage of potential items correctly performed, and algorithm generated grade and evaluators written comments on the learners performance (note all of these features are editable, based upon the users' needs)

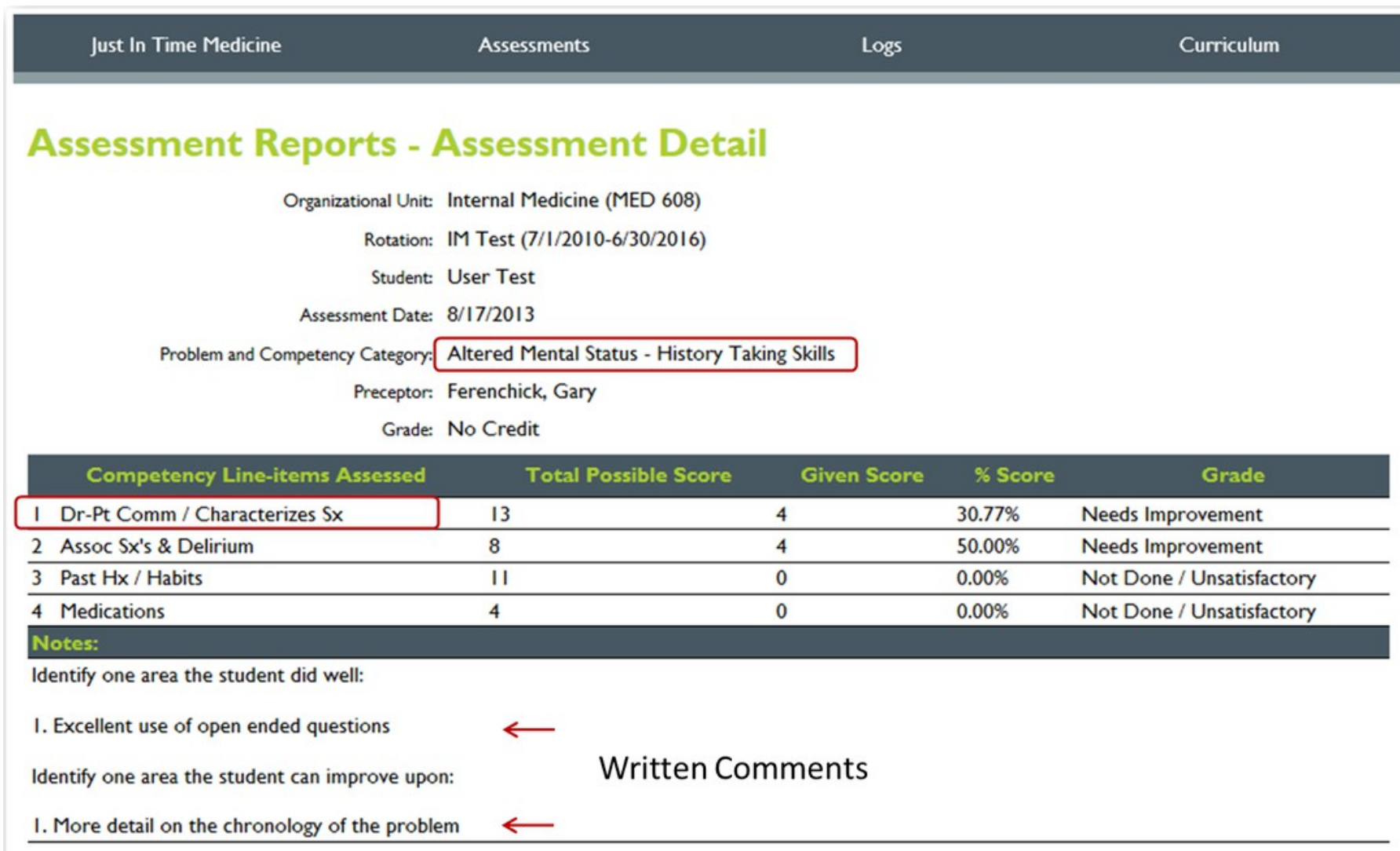




\section{Figure 6}

\section{JIT Detailed Cloud-based Reporting Options}

With the click of a hyperlink, a detailed list of all the items that were either performed or not by the student

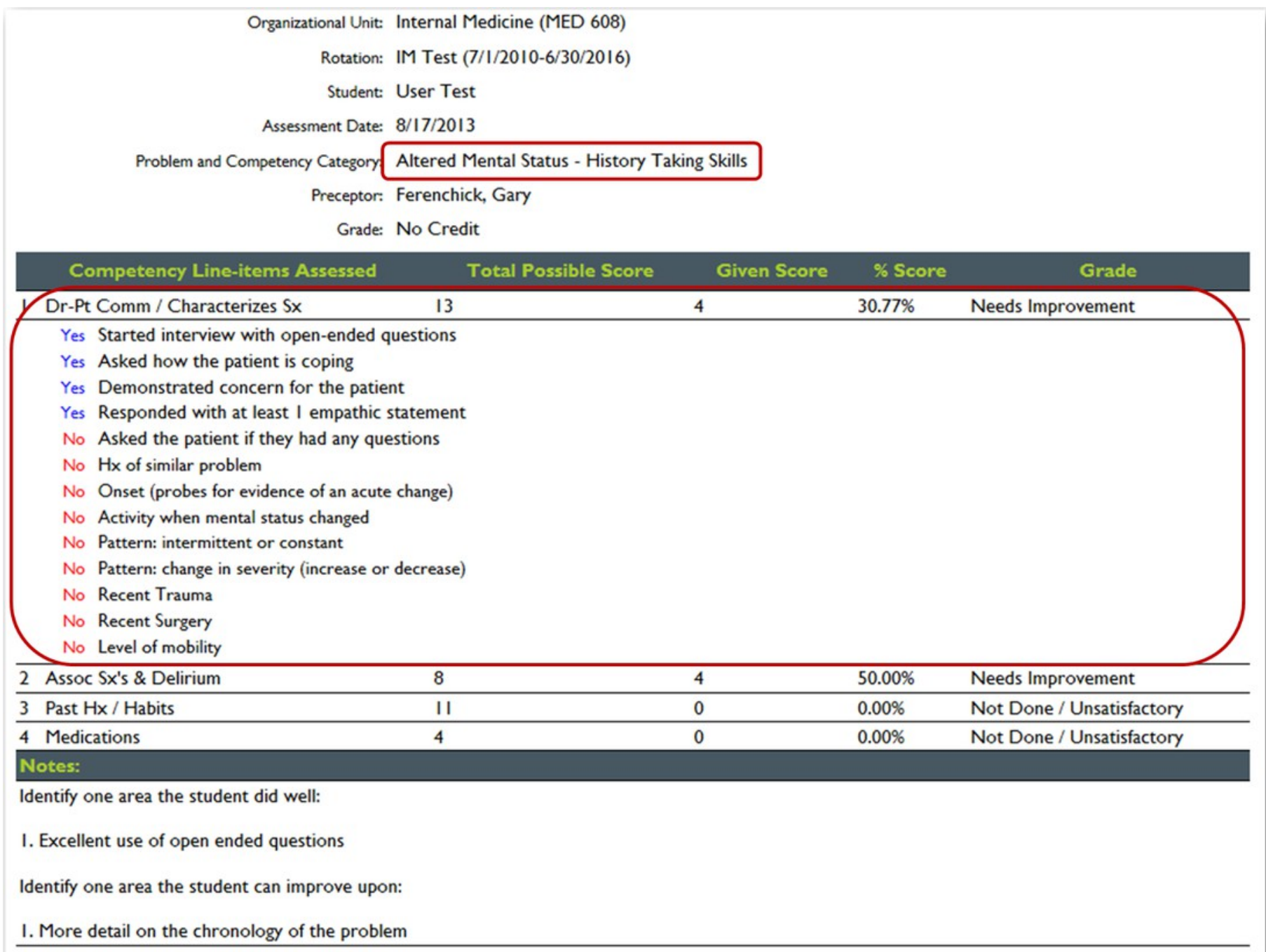




\section{Figure 7}

\section{JIT Cloud-Based Reporting Options}

Another option for a cloud-based record or registry of the learners performance. This image represents a milestone based report with the identified milestones (A); the milestone subcompetencies (B); a color-coded table of all of the learners assessments (C). A roll-over option (D) identifies which specific assessment is represented in each cell. This table shows the ACGME competency taxonomy for internal medicine.

\section{Taxonomy Assessment Reports - Summary}

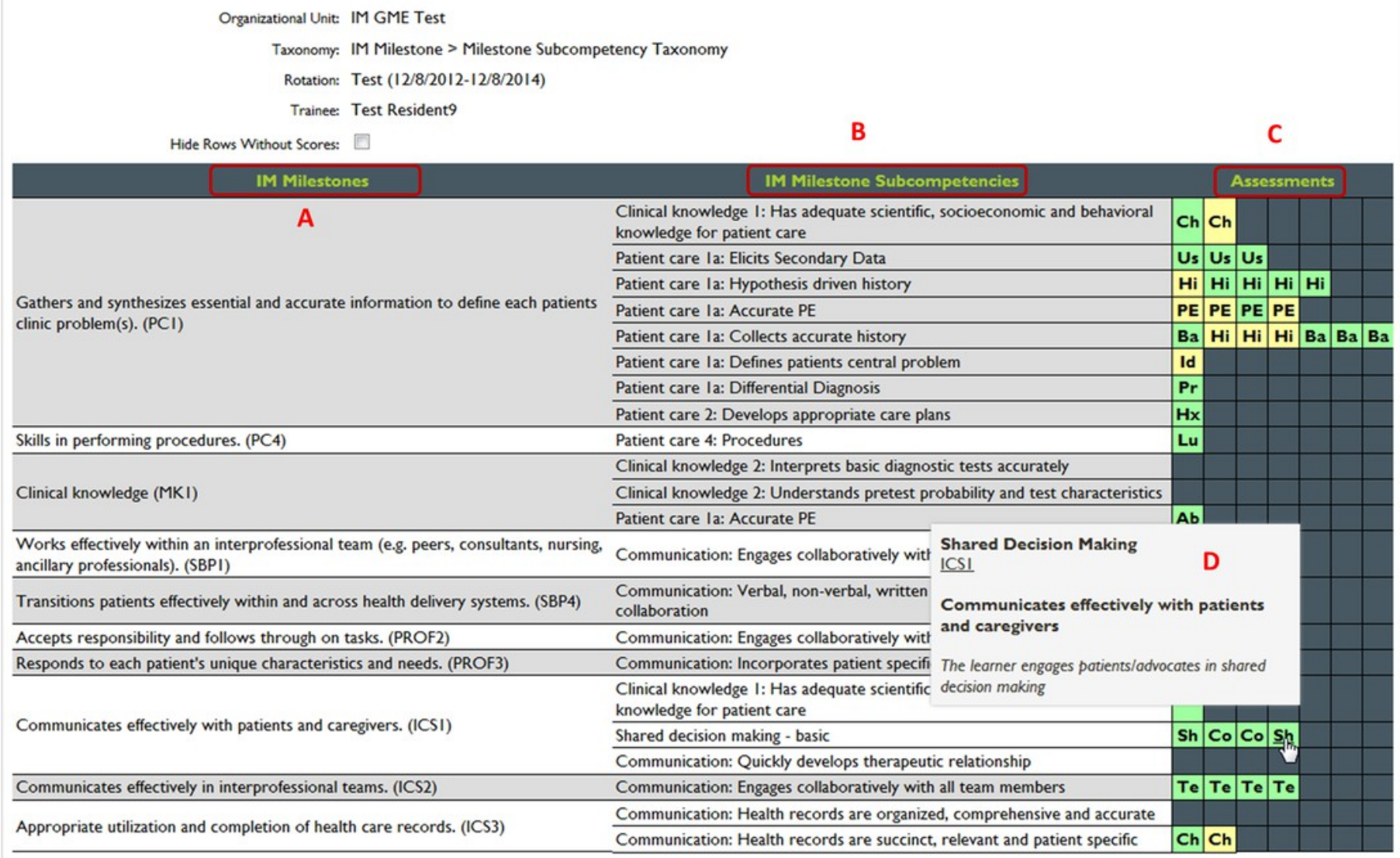




\section{Figure 8}

Bar chart of grade distribution comparing resident to faculty raters

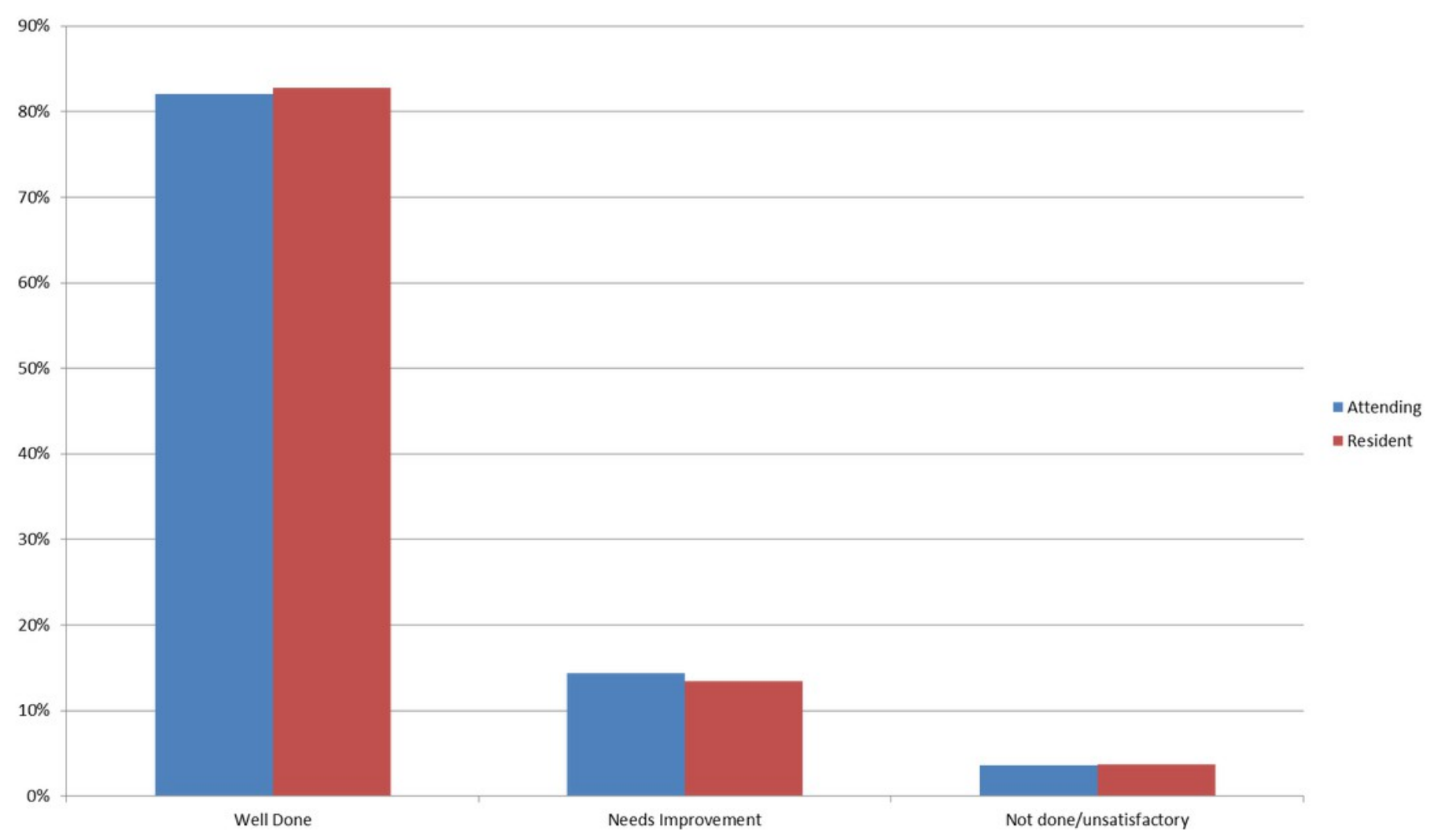

